\title{
Retinal pigment epithelial detachments in the elderly: classification and outcome
}

\author{
A G CASSWELL, D KOHEN, AND A C BIRD \\ From Moorfields Eye Hospital, City Road, London EC1V 2PD
}

SUMmARY Sixty-four eyes of 57 elderly patients with pigment epithelial detachments (PEDs) were studied with the aim of describing their morphological features and identifying prognostic factors. They were classified into four groups according to the following characteristics: (a) early fluorescence, (b) late fluorescence, (c) shallow detachment with limited fluorescence ('drusen type'), (d) irregular fluorescence. The following conclusions were drawn: (1) $30 \%$ developed demonstrable subretinal new vessels; all groups except the drusen type were susceptible, the irregular group being particularly prone. (2) $10 \%$ developed retinal pigment epithelial tears, and these occurred almost exclusively in the slow fluorescent group. (3) Flattening of PEDs was a feature of drusen type and early fluorescent groups. (4) Most patients lost vision. RPE tears occurred within a few months of presentation with immediate loss of vision; likewise rapid loss of vision followed the development of demonstrable new vessels, though not necessarily in those with changes thought to imply the presence of new vessels. Flattening after prolonged detachment was associated with pigment epithelial atrophy and invariable loss of vision. Visual acuity was maintained consistently only in those eyes with persistent detachment.

Pigment epithelial detachments (PEDs) are discrete, serous elevations of the retinal pigment epithelium. During fluorescein angiography the subpigment epithelial space fills progressively with dye and remains hyperfluorescent long after dye transit. ${ }^{1}$ Recent behavioural studies have shown that the visual prognosis may be determined by the size of the lesion and the age of the patient. Lewis ${ }^{2}$ has shown that in younger patients (less than 55 years old) the visual prognosis is good. Meredith et al. ${ }^{3}$ found that elderly patients with small PEDs have a relatively better visual prognosis than those with large lesions but identified no other features as useful indicators of prognosis.

PEDs have a complex variety of appearances. The nature of the different characteristics and the significance of particular changes to visual prognosis have not been defined. In particular it is acknowledged that the exclusion of subretinal new vessels can be difficult, so that it may be impossible to be sure that a lesion is 'avascular.' It has been considered that their

Correspondence to Mr A G Casswell, FRCS. presence may be implied by the presence of focal hyperfluorescent spots within a PED, yellow subretinal deposits, and turbid fluid.

In the past it has been thought that PEDs caused subretinal new vessels to develop and that this complication represented the principal threat to vision. Photocoagulation has been used to flatten the pigment epithelium with the aim of restoring vision and preventing the development of disciform lesions, but a recent controlled trial of elderly patients treated with argon laser photocoagulation implies that this form of therapy confers no visual benefit. ${ }^{4}$ During this trial sight threatening complications other than the development of subretinal new vessels were identified.

It was the results of the trial and the observations made during the study which stimulated the present review to classify the morphological features of PEDs and to identify whether any particular characteristics had prognostic significance. Information of this type may help in identifying the expectations for vision in a patient and would also contribute to planning any further trials of treatment for PEDs. 


\section{Material and methods}

We undertook a retrospective study of 64 consecutive untreated patients older than 55 years with pigment epithelial detachments who were referred to the Retinal Diagnostic Department at Moorfields Eye Hospital from 1976 to 1981 .

The criteria for the diagnosis of a PED was elevation of the retinal pigment epithelium with accumulation of fluorescein dye in the subpigment epithelial space during angiography. Patients with identifiable subretinal new vessels, subretinal haemorrhage, unexplained focal hyperfluorescent spots, or extensive subretinal exudates were excluded. Those patients with high myopia and any additional past or present eye disease which could affect the visual acuity were also excluded.

The following were recorded: the age and sex of the patient, duration of symptoms, corrected visual acuity, signs of age related macular changes (drusen etc.), the size, location, and ophthalmoscopic features of the PEDs, together with the pattern of fluorescence during angiography. At follow-up visits the visual acuity was recorded and colour photographs and fluorescein angiography were repeated.

In the first part of the study we classified foveal and juxtafoveal PEDs on the basis of their ophthalmoscopic and fluorescein appearances. In the second part we recorded the outcome and visual acuity of all PEDs that had been observed for at least one year, or less if they developed a sight losing complication within that time. All those patients who did not have adequate follow-up were requested to attend again.

The diagnosis of a disciform lesion was based on the demonstration of new vessels or the presence of subretinal haemorrhage in the absence of a tear of the retinal pigment epithelium (RPE). The development of yellow subretinal exudates and the delayed appearance of fluorescence was not regarded as diagnostic of subretinal neovascularisation.

\section{Results}

\section{CLASSIFICATION}

We classified 64 PEDs of 57 patients into four groups based on the fundal appearance and fluorescein angiograms.

Hyperpigmentation of the pigment epithelium (pigment figure) was a feature of many lesions and was not used to differentiate one group from another. In several lesions a patch of hypofluorescence was identified which appeared to be due to obstruction of fluorescein at the level of the pigment epithelium without any corresponding lesions seen by ophthalmoscopy (Fig. 1).

1. Early hyperfluorescent group (17 eyes). A

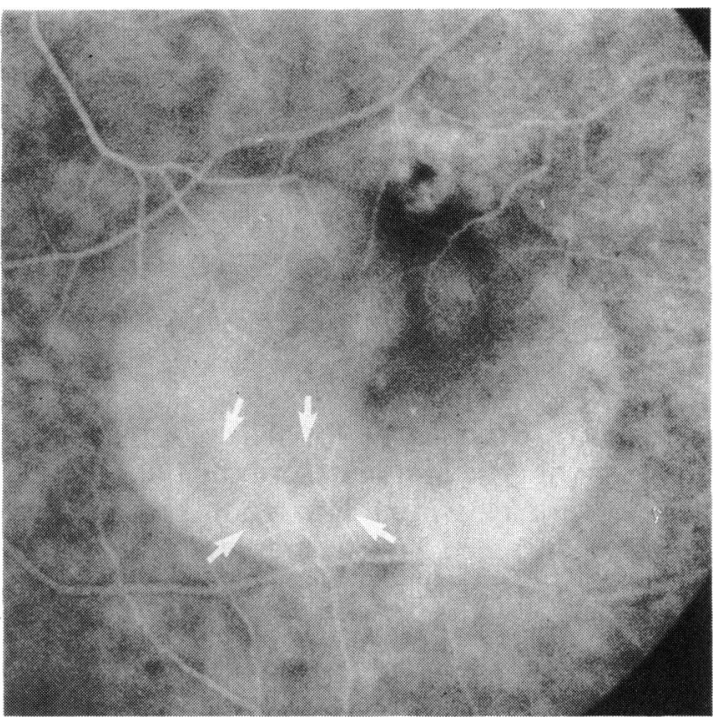

Fig. 1 Fluorescein angiography of a pigment epithelial detachment showing hypofluorescent patches in its lower part (arrows). No ophthalmoscopic abnormalities were found to correspond with these.

discrete dome-shaped elevation of the RPE is seen ophthalmoscopically. During fluorescein angiography the subpigment epithelial space fluoresces early and progresses to an even hyperfluorescence in the later stages of the transit (Figs. 1, 2). This appearance may be modified by small areas of

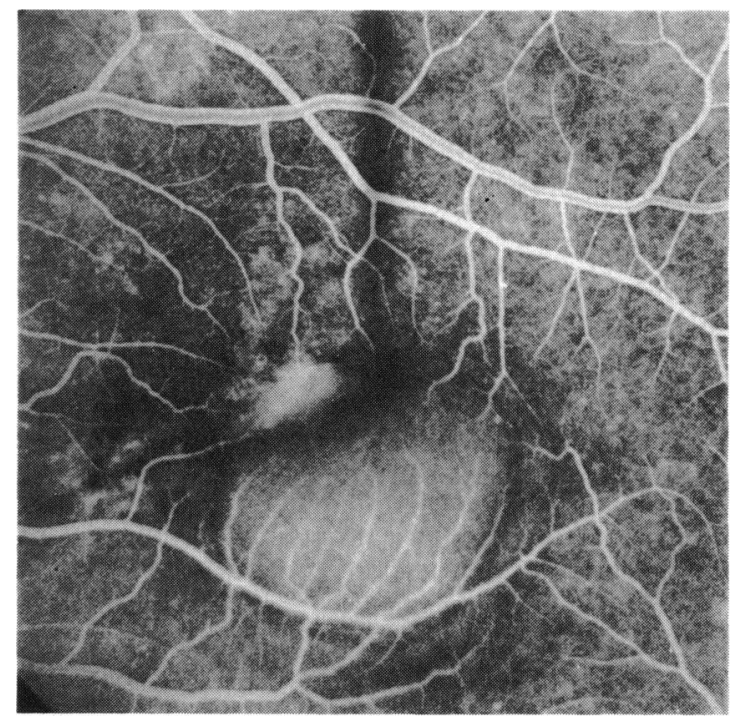

Fig. 2 Fluorescein angiography of a pigment epithelial detachment filling early in the angiogram with even hyperfluorescence. 
hyperfluorescence which can be identified as corresponding to areas of pigment epithelial atrophy or drusen that have become detached with the pigment epithelium or occasionally round well-defined zones of hypofluorescence that have no corresponding feature ophthalmoscopically.

2. Late fluorescing group (14 eyes). Ophthalmoscopically these cannot be differentiated from the early hyperfluorescent PEDs. They are characterised by the appearance of fluorescence after the arteriovenous phase (delayed appearance), which progresses slowly to an even and featureless hyperfluorescence (Figs. 3, 4). In some cases fluorescence may appear first at the edges of the PED and spread concentrically inwards.

3. Drusen type (18 eyes). Multiple large confluent drusen are seen in and surrounding these PEDs, which are usually shallow and irregular in outline. On fluorescein angiography they fluoresce faintly during the transit and do not progress to the bright hyperfluorescence seen in the lesions of other groups (Fig. $5)$. The presence of underlying drusen modified the angiographic appearance of the lesion in that at the site of the drusen the fluorescence was less marked than elsewhere in the lesion.

4. Irregular fluorescence (10 eyes). Though ophthalmoscopically indistinguishable from the first two groups, angiography reveals an overall fine irregularity of fluorescence. Typically the irregularity of fluorescence comprises multiple areas of hypo- and hyperfluorescence over the whole detachment. The

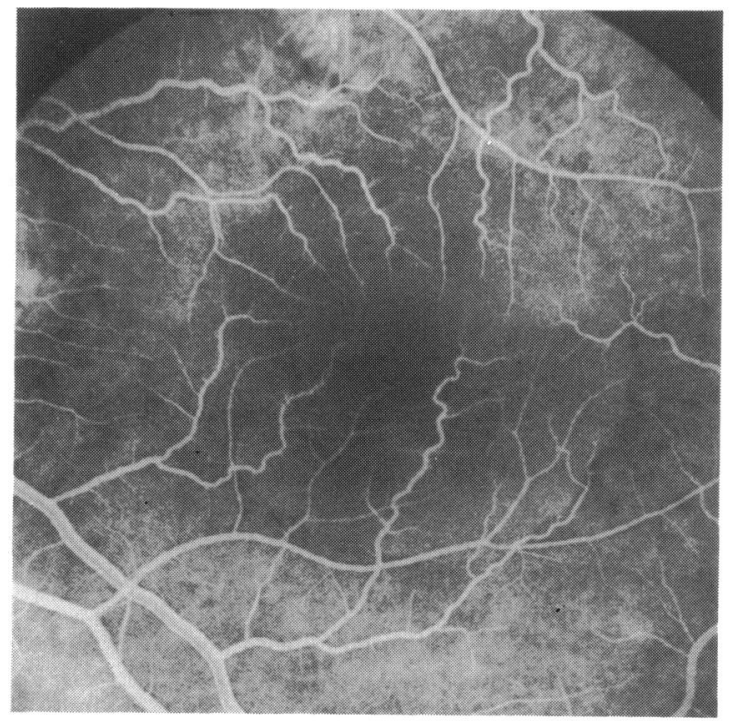

Fig. 3A hyperfluorescent areas increase in fluorescence during the study, whereas the hypofluorescent areas remain unchanged (Fig. 6).

5. Mixed group (5 eyes). There were five PEDs with two zones of different characteristics during angiography, usually one area of irregular fluorescence adjacent to a slowly filling hyperfluorescent area. Typically the half with slow fluorescence was the more elevated of the two.

Other characteristics did not serve to differentiate between groups, the average age, age ranges, and presenting visual acuities being similar (Table 1). Although size was variable within each group, there was a tendency for the Drusen PEDs to be smaller (0.96 disc diameters, Fig. 5) and for the slowly filling PEDs to be larger (2.1 disc diameters, Fig. 4).

\section{OUTCOME}

Morphological

A variety of changes were identified during the period of review (Table 2). With few exceptions spontaneous flattening occurred only after an interval of several years. In many there was persistence of the PED, though the characteristics changed in some. One drusen PED enlarged and became rapidly fluorescent, and several PEDs became irregularly fluorescent before either flattening or developing new vessels. In four eyes subretinal exudates developed round the margins of the detachment without demonstrable subretinal new vessels at any stage. In $30 \%$ a disciform lesion developed. In $10 \%$

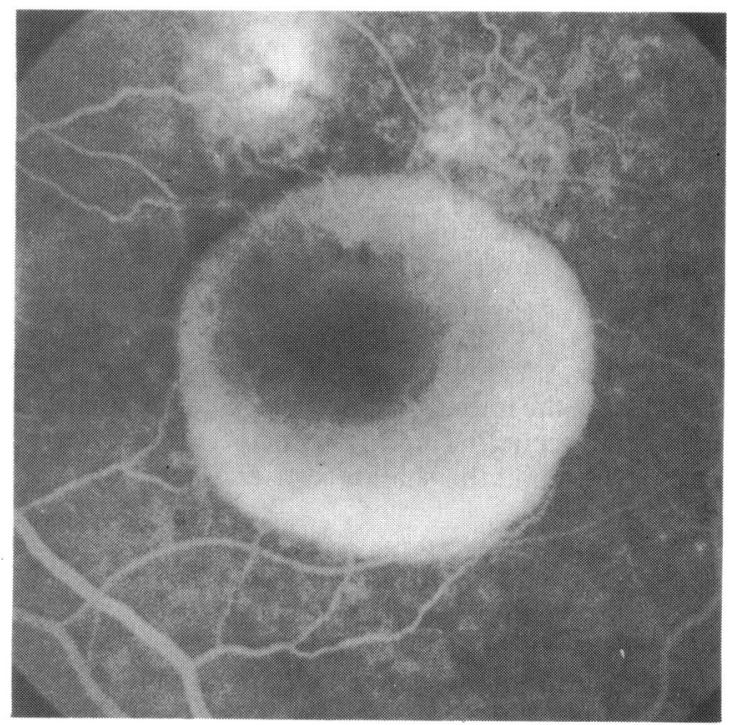

Fig. 3B

Fig. 3 Fluorescein angiography of a pigment epithelial detachment in the posterior pole showing slow development of hyperfluorescence, which is uniform. 


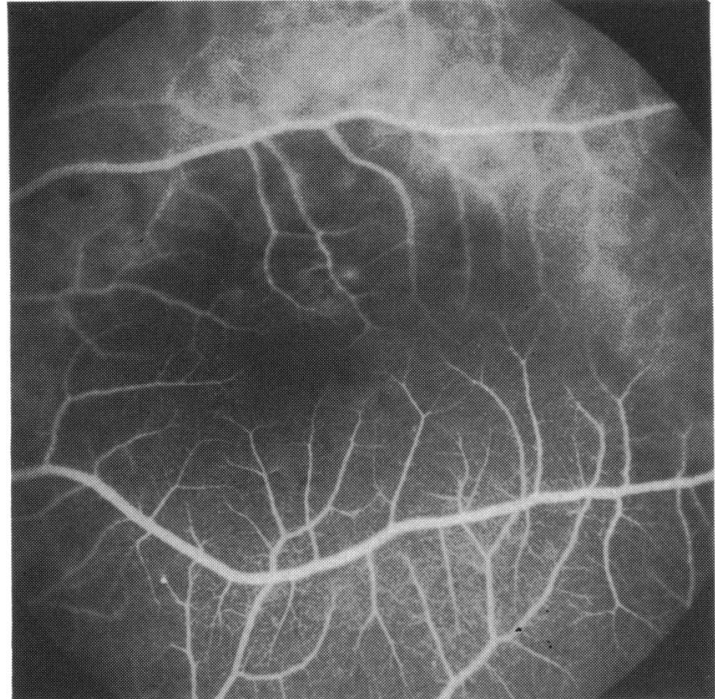

Fig. 4A

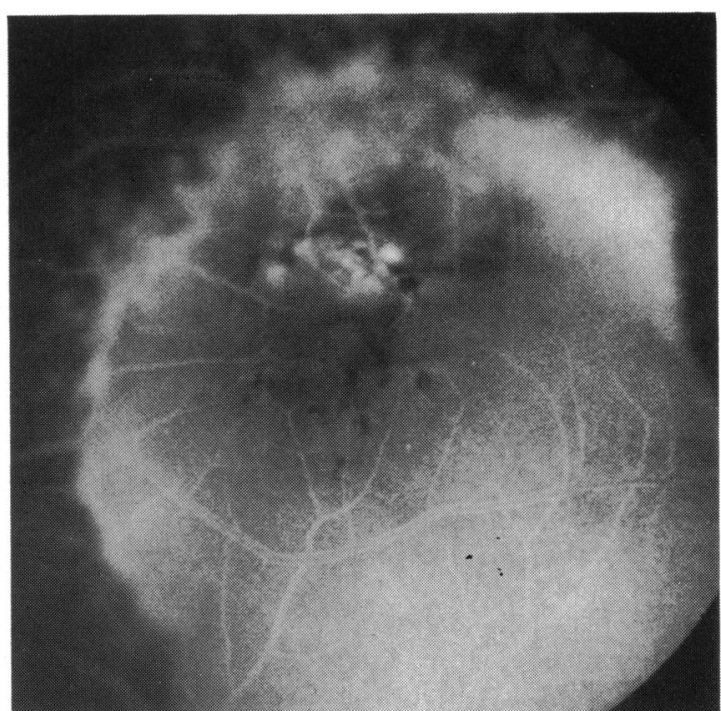

Fig. 4B

Fig. 4 Fluorescein angiography of a large pigment epithelial detachment showing slow acquisition of hyperfluorescence, which is uniform.

there was a tear of the pigment epithelium, and in each case this occurred within a few months of presentation. The true figure is probably higher than $10 \%$, as some patients presented with this early complication and were excluded from the review.

The characteristics of the lesion when the patient was first seen were not an absolute determinant of subsequent behaviour, but certain complications were clearly more common in some types than others (Table 2). Subretinal neovascularisation was much more common in those with fine irregular hyperfluorescence than in others, though this process became evident in all types except the shallow 'drusen detachments.' All but one of the tears occurred in 'slow hyperfluorescent' lesions.

\section{Visual}

The visual outcome was also correlated to some extent with the initial classification. The irregular and slow fluorescent PEDs had worse final vision than the other types, and the shallow drusen types fared best of the groups.

As might have been predicted, the final visual acuity was poor in those patients who developed tears of the retinal pigment epithelium or subretinal new vessels (Table 3 ). The visual outcome was also bad in those lesions in which there was spontaneous flattening of the pigment epithelium. The only lesions associated with consistently good vision at the end of the review period were those with persistent detachment.

\section{Discussion}

INTERPRETATION OF APPEARANCE

Certain determinants of behaviour of PEDs during fluorescein angiography might be predicted on the basis of theoretical considerations together with the limited knowledge of the structural changes occurring within the lesion derived from histopathological studies. It is generally assumed that the hyperfluorescence of PEDs is due to the increased thickness of the fluorescing medium due to dye accumulation in the subpigment epithelial space, the fluorescein being derived from the choroid having passed into subretinal space by diffusion through Bruch's membrane. Unequal rates of dye accumulation might be predicted if there were variation of the lipid content within Bruch's membrane or the subepithelial fluid from one lesion to another or within a single lesion. This would influence the speed of diffusion, since fluorescein-sodium is highly soluble in water but not in lipid. It is well recognised that material accumulating within and on the inner surface of Bruch's membrane with age contains both lipid and protein, but the results of a systematic analysis of this material in man do not exist.

The appearance of fluorescence may also be modified by the transparency of the subpigment epithelial fluid and the detached structures; this is well illustrated by the light absorption by luteal pigment and pigment figures. Clinical evidence implies that pigment epithelium may become detached, with drusen 


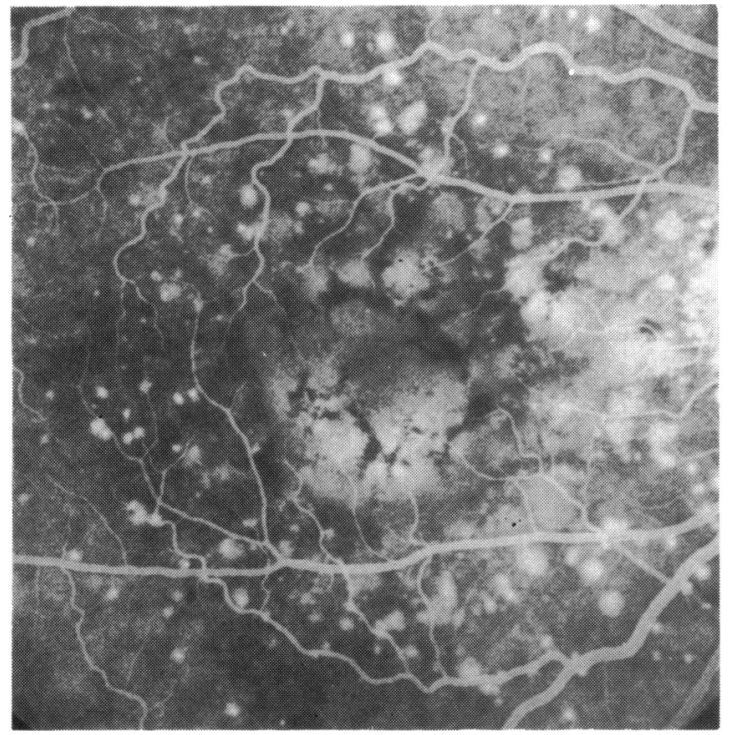

Fig. 5 Fluorescein angiography of a shallow pigment epithelial detachment associated with drusen.

on its under surface in some lesions but not in others. That there should be a similar disparity in the behaviour of pigment epithelial basement membrane has been postulated by Hoskin et al. ${ }^{5}$ Such variation in the distribution of material would predictably influence the appearance of the lesion clinically and during angiography, and this could account for the two different zones of a 'mixed PED.'

The slow appearance of fluorescence in some lesions may be accounted for by slow diffusion of dye into the subretinal space due to high lipid content within the subpigment epithelial fluid or in Bruch's membrane, or by the presence of blue/green absorbing material within the subpigment epithelial fluid, so that fluorescence is not recorded until the dye has diffused as far as the detached pigment epithelium. The latter would explain the appearance of fluorescein at the periphery of the lesion in the early stages of the study, a feature common to many lesions. These two situations may coexist, since lipofuscin, which absorbs blue light well, is the most likely lipid

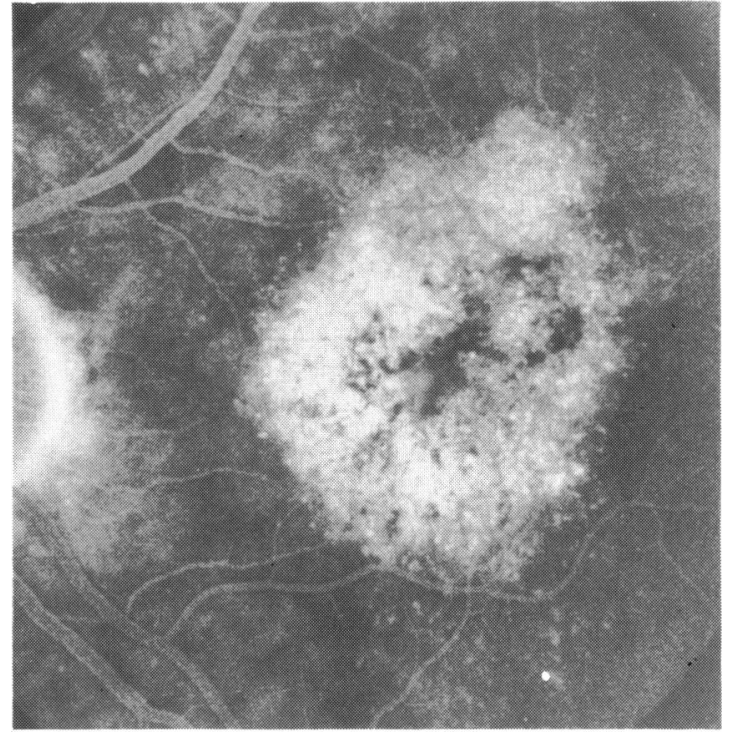

Fig. 6 Fluorescein angiography of a pigment epithelial detachment with irregular hyperfluorescence.

to accumulate at these sites. By contrast in those PEDs which become rapidly and evenly fluorescent it is unlikely that there is a high lipid content within either Bruch's membrane or the subretinal fluid or that the detached tissues contain large amounts of blue/green absorbing pigments.

The diffuse irregular fluorescence in the irregular masking group suggests irregular transmission of light by the detached tissues due to material on the outer aspect of the detached retinal pigment epithelium. While the origin of such a material is unknown, it could result from deposition of phagosomal debris, thickening of pigment epithelial basement membrane, or possibly the product of undetected new vessels.

The focal and increasing fluorescence which corresponds with large drusen seen on the undersurface of Bruch's membrane is probably due to displacement of pigment within the detached pigment epithelium and accumulation of dye in the drusen. The areas of hypofluorescence seen without

Table 1 Pigment epithelial detachments at presentation

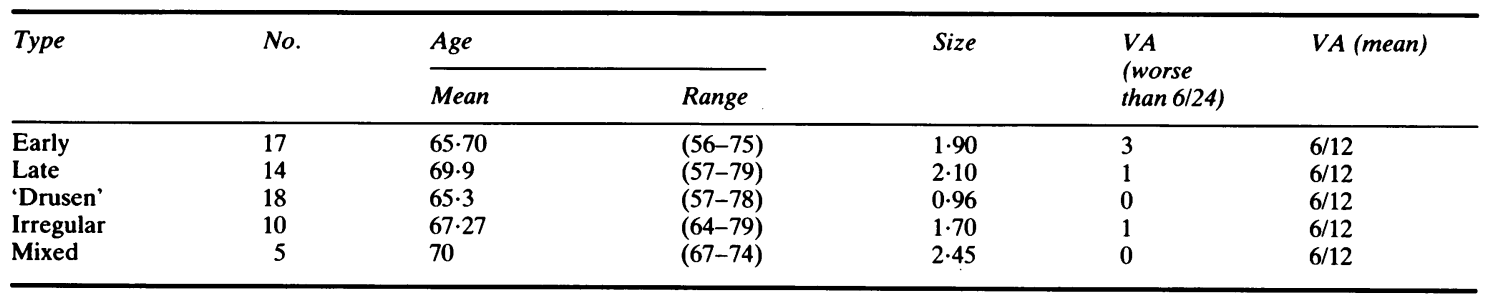

Size measured in disc diameters. 
Table 2 Outcome of pigment epithelial detachments with a follow-up of one year or more

\begin{tabular}{|c|c|c|c|c|c|c|c|}
\hline \multirow[t]{2}{*}{ Type } & \multirow[t]{2}{*}{ No. } & \multicolumn{4}{|c|}{ Outcome } & \multirow{2}{*}{$\begin{array}{l}V A \\
\text { (worse } \\
\text { than 6/24) }\end{array}$} & \multirow[t]{2}{*}{$V A$ (mean) } \\
\hline & & Flat & $P E D$ & Disc & Tear & & \\
\hline Typical & 16 & 3 & 6 & 6 & 1 & 9 & $6 / 24$ \\
\hline Late & 11 & 0 & 4 & 3 & 4 & 5 & $6 / 36$ \\
\hline 'Drusen' & 15 & 6 & 9 & 0 & 0 & 5 & $6 / 12$ \\
\hline Irregular & 8 & 0 & 2 & 6 & 0 & 4 & $6 / 48$ \\
\hline
\end{tabular}

Flat $=$ PEDs that spontaneously flatten.

PED=pigment epithelium remaining detached.

Disc $=$ development of a disciform lesion.

Tear=pigment epithelial tear.

Table 3 Visual change of PEDs in terms of their outcome

\begin{tabular}{lclllll}
\hline Outcome & No. & Age & $\begin{array}{l}\text { Size } \\
(D D)\end{array}$ & $\begin{array}{l}\text { Initial VA } \\
\text { (mean) }\end{array}$ & $\begin{array}{l}\text { Follow-up } \\
\text { (months) }\end{array}$ & $\begin{array}{l}\text { Final VA } \\
\text { (mean) }\end{array}$ \\
\hline Flat & 9 & 64 & $1 \cdot 5$ & $6 / 13$ & 49 & $6 / 30$ \\
PED & 21 & $65 \cdot 1$ & $1 \cdot 23$ & $6 / 10$ & 6 & 6 \\
Disciform & 15 & $67 \cdot 4$ & $2 \cdot 25$ & $6 / 13$ & 6 & 6 \\
Tears & 6 & 70 & $3 \cdot 04$ & $6 / 21$ & 7 & 6 \\
\hline
\end{tabular}

Follow-up=mean time between presentation and event. $\mathrm{DD}=$ disc diameter.

corresponding ophthalmoscopic changes appear to be due to transmission change at the level of the pigment epithelium, which might be caused by accumulation of a blue/green absorbing pigment other than melanin, which may be intra- or extracellular. Collections of lipofuscin-containing macrophages or RPE cells growing on the outer surface of the detached RPE would account for such an appearance.

Drusen PEDs are small, shallow, and surrounded by and containing large drusen. The large drusen contained within the PED fluoresce faintly towards the end of the transit, and because they occupy most of the sub-RPE space there is relatively little subRPE fluid to accumulate fluorescein, thus limiting fluorescence. The difficulty differentiating some of these ophthalmoscopically from confluent drusen suggests that they may develop as a direct result of accumulation of confluent drusen rather than indicating the onset of a new process.

\section{OUTCOME}

If the variation in the appearance of retinal pigment epithelial detachments is due to different distribution of cellular and non-cellular material within the lesion, correlation might be expected between the clinical characteristics of the lesion and its subsequent behaviour.

Hoskin has postulated that the features of 'slow filling' lesions implied that the retinal pigment epithelium may be detached without a normal basement membrane and that the lack of physical support within the detached tissue would increase the risk of tearing. The results of this study support the conclusion concerning the risk of this complication occurring in this type of lesion, though the conclusions concerning pathogenesis have not been tested. The larger size of these lesions may also be important in the pathogenesis of the tear. While disciform lesions occurred within this group, four patients had no evidence of subretinal new vessels at the end of the review period. This means that turbidity of the subpigment epithelial fluid does not necessarily imply the inevitable presence of neovascularisation. It is clear, however, that in such cases new vessels may have been present on the inner surface of Bruch's membrane when the patient was first seen and may not have been identified on angiography. This phenomenon has been identified in the past, ${ }^{4}$ and it was shown that blood vessels at this level may not involve the detached pigment epithelium and do not necessarily cause loss of visual acuity.

Disciform lesions were common in the lesions with finely irregular hyperfluorescence. This may be related to the presence of debris on the under surface of the detached tissues, which may stimulate neovascularisation and in particular cause the new vessels to grow at the level of the pigment epithelium rather on the inner surface of Bruch's membrane.

Few sight threatening complications were seen in the 'drusen RPE detachments.' This may be a consequence of their small size and the shallow 
detachment; there is little evidence that the large drusen would suppress the development of new vessels.

It was not surprising that severe visual loss was common in patients with tears of the retinal pigment epithelium or vascular disciform lesions. However, the explanation for the poor vision in those lesions which flattened spontaneously is not evident. It is possible that flattening caused function to be lost or that tissue failure caused both the loss of vision and the resolution of the detachment, the second explanation being the most attractive of the two.

Some useful prognostic information can be gained from the morphological appearance of PEDs. PEDs of all groups except the drusen group are at risk of developing disciform lesions, and those with irregular fluorescence were the worst affected. Nearly all the RPE tears were in the slow fluorescing group. The visual prognosis is poor for all groups. RPE tears are followed by visual loss within days of occurrence.
The development of new vessels was accompanied by visual loss within a few months, and those that flattened after several years had atrophy of the RPE and visual loss. These results should be useful in the planning of any future trial of the treatment of pigment epithelial detachments.

\section{References}

1 Donald J, Gass M. Pathogenesis of disciform detachment of the neuroepithelium. Am J Ophthalmol 1976; 63: 617-44.

2 Lewis ML. Idiopathic serous detachment of the pigment epithelium. Arch Ophthalmol 1978; 96: 620-4.

3 Meredith TA, Braley RE, Aaberg TM. Natural history of serous - detachments of the retinal pigment epithelium. Am J Ophthalmol 1979; 88: 643-51.

4 Moorfields Macular Study Group. Retinal pigment epithelial detachments in the elderly: a controlled trial of laser. $\mathrm{Br} J$ Ophthalmol 1982; 66: 1-16.

5 Hoskin A, Bird AC, Schmi K. Tears of the detached retinal pigment epithelium. Br J Ophthalmol 1981; 65: 417-22. 